Corresponding author: Richard.Wilson@

nationwidechildrens.org

(C) 2019 Hickey et al. This article is distributed under the terms of the Creative Commons Attribution-NonCommercial License, which permits reuse and redistribution, except for commercial purposes, provided that the original author and source are credited.

Ontology terms: aplasia/ hypoplasia of the patella; bilateral talipes equinovarus; congenital knee dislocation; joint laxity

Published by Cold Spring Harbor Laboratory Press

doi:10.1101/mcs.a004176

\section{Novel in-frame FLNB deletion causes Larsen syndrome in a three-generation pedigree}

\author{
Scott E. Hickey, ${ }^{1,2}$ Daniel C. Koboldt, ${ }^{1,3}$ Theresa Mihalic Mosher, ${ }^{1,2,3}$ \\ Patrick Brennan, ${ }^{3}$ Beth A. Schmalz, ${ }^{2}$ Erin Crist, ${ }^{3}$ Kim L. McBride, ${ }^{1,2,4}$ \\ Brent H. Adler, ${ }^{1}$ Peter White, ${ }^{1,2}$ and Richard K. Wilson ${ }^{1,2}$

\begin{abstract}
${ }^{1}$ Department of Pediatrics, The Ohio State University, Columbus, Ohio 43205, USA; ${ }^{2}$ Division of Genetic and Genomic Medicine, Nationwide Children's Hospital, Columbus, Ohio 43205, USA; ${ }^{3}$ Institute for Genomic Medicine at Nationwide Children's Hospital, Columbus, Ohio 43205, USA; ${ }^{4}$ Center for Cardiovascular Research, The Research Institute at Nationwide Children's Hospital, Columbus, Ohio 43205, USA
\end{abstract}

\begin{abstract}
A 4-yr-old female with congenital knee dislocations and joint laxity was noted to have a strong maternal family history comprising multiple individuals with knee problems and clubfeet. As the knee issues were the predominant clinical features, clinical testing included sequencing of $L M X 1 B, T B X 2$, and TBX4, which identified no significant variants. Research genome sequencing was performed in the proband, parents, and maternal grandfather. A heterozygous in-frame deletion in FLNB c. 5468_5470delAGG, which predicts p.(Glu1823del), segregated with the disease. The variant is rare in the gnomAD database, removes a residue that is evolutionarily conserved, and is predicted to alter protein length. Larsen syndrome may present with pathology that primarily involves one joint and thus may be difficult to differentiate clinically from other skeletal dysplasias or arthrogryposis syndromes. The p.(Glu1823del) variant maps to a filamin repeat domain where other disease-causing variants are clustered, consistent with a probable gain-of-function mechanism. It has reportedly been observed in two individuals in the gnomAD database, suggesting that mild presentations of Larsen syndrome, like the individual reported here, may be underdiagnosed in the general population.
\end{abstract}

[Supplemental material is available for this article.]

\section{CLINICAL PRESENTATION}

The proband was a 4-yr-old European-American female born weighing $7 \mathrm{lbs} 3 \mathrm{oz}$ at term by elective cesarean delivery due to breech presentation. She was born with her knees locked in extension and her feet held up against her head. She was subsequently found to have bilateral congenital knee dislocations (Supplemental Fig. 1), which were treated surgically beginning at 6 mo of age with staged open reductions and femoral shortenings. The mother was known to have also had clubfeet and knee dislocations requiring several orthopedic surgeries. Other family members are also noted to be affected as shown in the pedigree (Table 1; Fig. 1). The diagnosis of nail-patella syndrome (NPS) (OMIM 161200) was suspected by her orthopedic physician and agreed on by the initial clinical geneticist who evaluated the proband. $L M X 1 B$ sequencing and deletion/duplication analysis were negative.

The proband was reevaluated by a second geneticist at $2.5 \mathrm{yr}$ of age, and the clinical diagnosis of NPS was questioned because of emerging new clinical and developmental 


\begin{tabular}{lcccc}
\hline Table 1. Clinical features & & & & \\
\hline HPO term & Proband & Mother & Maternal uncle & Grandfather \\
\hline Knee dislocation & + & + & + & + \\
Talipes equinovarus & - & + & + & unk \\
Scoliosis & - & + & unk & unk \\
Bowing of the legs & - & - & + & - \\
Cleft palate & - & - & - & - \\
\hline
\end{tabular}

Human Phenotype Ontology (HPO) terms are listed with an indication of whether each patient was positive (+), negative $(-)$, or unknown (unk) for each feature.

information. A lateral knee $\mathrm{X}$ ray obtained at 20 mo demonstrated soft tissue in the region of the patella, suggesting the presence of a cartilaginous patella. A skeletal survey showed the normal appearance of the elbows, no vertebral segmentation defects or fusions, no iliac horns, and metatarsus adductus. The appearance of the humeri and metacarpals was normal (Supplemental Fig. 2). Bilateral knee ultrasound at 3 yr of age showed normal morphology of bilateral patellae with central ossification centers. Sequencing and deletion/duplication analysis of TBX2 and TBX4 were both performed clinically with no clinically significant findings. The family was subsequently enrolled for research genome sequencing.

At $4 \mathrm{yr}$ of age, the proband had normal stature (40th centile) and apparently normal speech and language development. She was noted to have a depressed nasal bridge and root, midface retrusion, mild telecanthus, and a pointed chin. The mother was noted to have similar facial features. The proband did not have cleft palate/bifid uvula, tracheomalacia, spatulate thumbs, abnormally long fingers, or cervical spine dislocation (although her occipital condyles were noted to be relatively large).

\section{TECHNICAL ANALYSIS AND METHODS}

Genome sequencing was performed on genomic DNA from the proband, mother, father, and maternal grandfather using an Illumina NovaSeq6000 instrument according to the manufacturer's protocols. Reads were mapped to the GRCh37 reference sequence and secondary data analysis was performed using Churchill (Kelly et al. 2015). The average sequence depth achieved was $\sim 30.6 \times$ for the blood samples (proband, mother, and father) and $23.6 \times$ for the saliva sample (grandfather); sequencing metrics are provided in Supplemental Table 1. Our general approach to variant annotation and prioritization has been described (Koboldt et al. 2018); for this evaluation, we prioritized rare nonsynonymous coding variants segregating with disease under a dominant inheritance model because of the family history (Supplemental Table 2).

\section{VARIANT INTERPRETATION}

We identified a heterozygous 3-bp deletion in FLNB (Table 2) in the proband and two affected family members (mother and maternal grandfather) that was absent from the unaffected father. It is predicted to cause an in-frame deletion of a single amino acid (glutamic acid) at position 1823 in the protein. Please see Supplemental Material for an explanation of variant classification nomenclature (i.e., PM2, PP1). The variant is present in dbSNP (rs1470699812) and reportedly was observed in two non-Finnish European individuals in the gnomAD database; however, supporting read data are available for only one of these carriers. We 


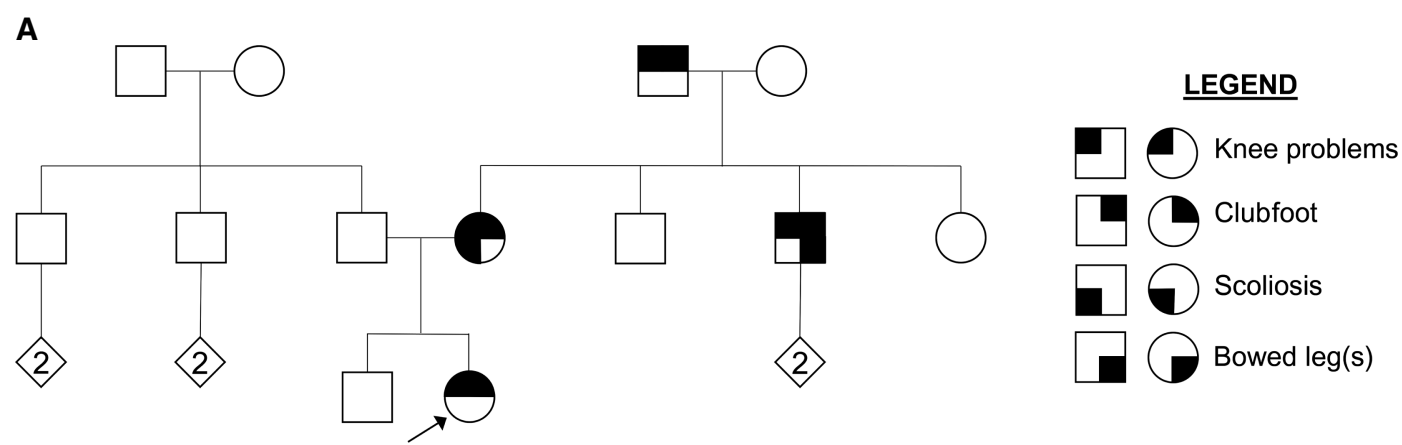

B

FLNB Variant in Family RD180047

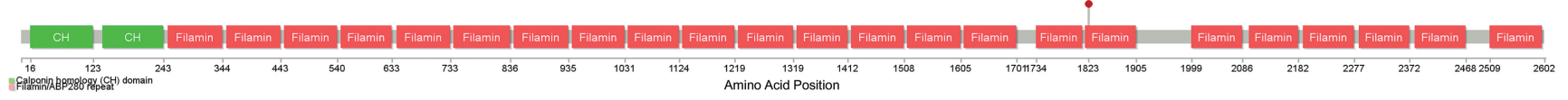

FLNB Variants Causing Larsen Syndrome
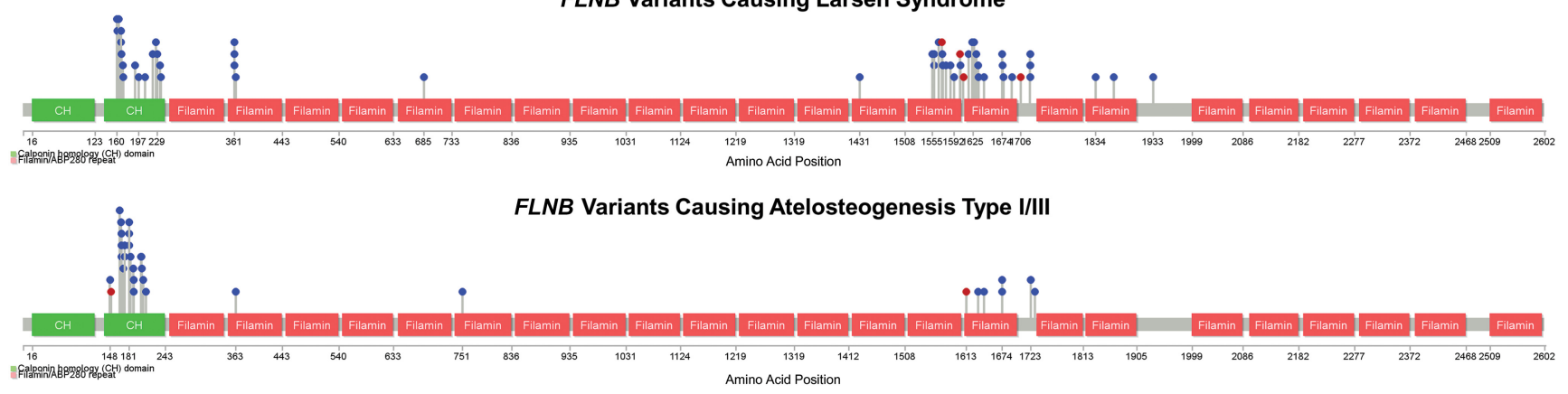

FLNB Variants Causing Boomerang Dysplasia

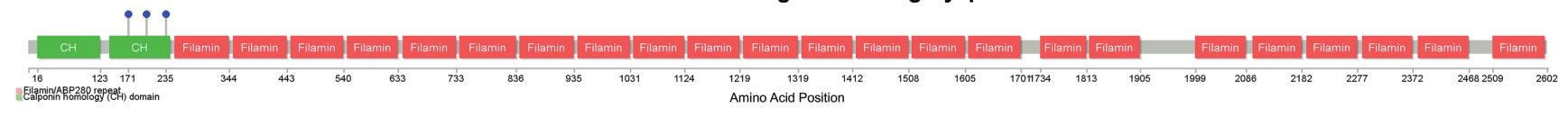

\begin{tabular}{|c|c|c|c|c|c|}
\hline \multicolumn{6}{|c|}{ LEGEND } \\
\hline $\mathrm{CH}$ & Calponin homology domain & Filamin & Filamin repeat domain & Missense variant & In-frame variant \\
\hline
\end{tabular}

Figure 1. Pedigree and FLNB variant. (A) Pedigree for RD180047 depicting the presence of cardinal features, namely knee problems, clubfoot, scoliosis, and bowed lower leg(s). (B) Graphical representation of FLNB variants and domain structures. The variant in family RD180057 is shown (top plot) in comparison to reported disease-causing variants for Larsen syndrome (second from top plot), atelosteogenesis type I/III (third from top plot), and boomerang dysplasia (bottom plot). Variants are plotted by amino acid position based on the canonical transcript NM_001164317.1. Missense variants are shown in blue; in-frame deletions are shown in red. Disease-causing variants were obtained from ClinVar (20190424), HGMD (20170720), and literature reports. Only variants reported for autosomal dominant OMIM conditions are shown. Plots were generated using Lollipops v1.3.2 using UniProt ID \#O75369. Domains were retrieved using the Pfam API.

therefore estimate its allele frequency at 1-2 per 125,000 individuals. It has not been reported in other public cohorts nor has it been submitted to ClinVar. Although the phenotypic status of gnomAD individuals is not available, if they are presumed to be healthy, then a conservative application of ACMG guidelines would not apply PM2 (absent from controls).

Although the variant occurs in a filamin repeat domain, the local sequence context is not repetitive, suggesting that this variant alters protein length and may have a deleterious impact (PM4). It segregates with disease in this family (PP1) and removes a residue that is 
Table 2. Genomic findings and variant interpretation

\begin{tabular}{cccccc}
\hline Gene & Genomic location & HGVS cDNA & HGVS protein & Zygosity/parent of origin & Interpretation \\
\hline FLNB & $\begin{array}{c}\text { Chr 3:58129288 } \\
\text { GAG/- (hg19) }\end{array}$ & NM_001164317.1:c. 5468_5470delAGG & p.(Glu1823del) & Het/Mat & VUS (PM4, PP1)
\end{tabular}

Genomic coordinates reflect build GRCh37 (hg19).

evolutionary conserved across vertebrate species. It also occurs in the 17th filamin repeat of FLNB near other pathogenic variants that have been reported in individuals with autosomal dominant Larsen syndrome (OMIM 150250) (Fig. 1B). Under ACMG guidelines, this variant is assessed as a variant of uncertain significance (VUS; Table 2), but we believe it is likely the cause of the patient's disease.

\section{SUMMARY}

Larsen syndrome (OMIM 150250) was first described in 1950 in a clinical series of six patients with multiple congenital joint dislocations in association with particular dysmorphic facial features, equinovarus foot deformities, and occasionally cleft palate or other skeletal anomalies (Larsen et al. 1950). Heterozygous missense variants in FLNB were originally described in four simplex affecteds and one family with autosomal dominant inheritance of Larsen syndrome (Krakow et al. 2004). Subsequent authors have described Larsen syndrome as a clinically and radiographically characteristic disorder (Girisha et al. 2016). The distribution of disease-causing variants is nonrandom; most variants reported for Larsen syndrome cluster in the actin-binding domain (ABD) and filamin repeats 13-17 (Bicknell et al. 2007). Filamin proteins are large cytoplasmic actin-binding proteins that regulate the cytoskeletal network (Stossel et al. 2001). Filamins contain an amino-terminal ABD, up to $24 \mathrm{lg}$-like folds interrupted by two "hinge" regions, and a filamin repeat at the carboxyl terminus (Girisha et al. 2016). The proteins are highly similar and can interact to form homo- and heterodimers. Mouse FLNB knockout models recapitulate the human knockout phenotype (distinct from Larsen syndrome as described below) with skeletal malformations including vertebral anomalies, shortened distal limbs, and spinal curvature (Lu et al. 2007; Zhou et al. 2007).

FLNB-related disorders associated with heterozygous variants and autosomal dominant inheritance represent a continuous spectrum of severity with atelosteogenesis type 1 (OMIM 108720) and boomerang dysplasia (OMIM 112310) representing the severe end and Larsen syndrome the mild end (Robertson 2017). Homozygous or compound heterozygous loss-offunction variants are associated with spondylocarpotarsal synostosis (STS) (OMIM 272460), a skeletal dysplasia characterized by disproportionate short stature and carpal and tarsal synostosis (Fig. 1B, bottom). There is limited understanding of the pathophysiology of abnormal FLNB function in Larsen syndrome, and the genotype-phenotype relationship remains unclear. Variants reported to cause Larsen syndrome are predominantly missense variants or, less commonly as in the individual described here, in-frame deletions (Fig. 1B). They appear to cluster in annotated domains near the amino-terminal or in the middle of the protein, consistent with a gain-of-function mechanism (Sawyer et al. 2009). Unlike the loss-of-function variants reported in STS, pathogenic variants associated with Larsen syndrome do not reduce the actin-binding affinity of FLNB, suggesting that they may instead exert a more general effect on targeting of filamin B to the appropriate actin structures (Sawyer et al. 2009). Enhanced actin binding and bundling as well as dysregulation the function of hinge 1 are two proposed mechanisms (Daniel et al. 2012). 
In summary, a heterozygous 3-bp deletion in FLNB was found in a child and her mother with clinical features consistent with Larsen syndrome. The variant also segregated in a maternal grandfather who was described by history to have features consistent with Larsen syndrome. Although classified as a VUS, we argue that the variant is causative of the phenotype in this family based on the fit of the clinical phenotype and the rationale provided in the Variant Interpretation section. As the clinical phenotype may be mild and this variant was reportedly observed in two individuals in the gnomAD database, there may be individuals in the general population with Larsen syndrome related to this variant who go clinically undiagnosed.

\section{ADDITIONAL INFORMATION}

\section{Data Deposition and Access}

The variants and their interpretations have been deposited in the ClinVar database (https:// www.ncbi.nlm.nih.gov/clinvar/) under accession number SCV000599274.

\section{Ethics Statement}

Written consent was obtained enrolling subjects into a research protocol approved by the Institutional Review Board at Nationwide Children's Hospital (IRB11-00215 Study: Using Genome Sequencing to Identify Causes of Rare Birth Defects and Rare Disorders).

\section{Acknowledgments}

We thank the patient and her family for participation in this research. We thank April Lehman, $M D$, for patient clinical care and clarification of family history.

Competing Interest Statement The authors have declared no competing interest.

Received March 14, 2019; accepted in revised form August 23, 2019.

\section{Author Contributions}

All authors contributed to scientific discussion, variant interpretation, and manuscript review.

\section{Funding}

This work was supported by The Research Institute at Nationwide Children's Hospital.

\section{REFERENCES}

Bicknell LS, Farrington-Rock C, Shafeghati Y, Rump P, Alanay Y, Alembik Y, Al-Madani N, Firth H, Karimi-Nejad $\mathrm{MH}$, Kim CA, et al. 2007. A molecular and clinical study of Larsen syndrome caused by mutations in FLNB. J Med Genet 44: 89-98. doi:10.1136/jmg.2006.043687

Daniel PB, Morgan T, Alanay Y, Bijlsma E, Cho TJ, Cole T, Collins F, David A, Devriendt K, Faivre L, et al. 2012. Disease-associated mutations in the actin-binding domain of filamin B cause cytoplasmic focal accumulations correlating with disease severity. Hum Mutat 33: 665-673. doi:10.1002/humu.22012

Girisha KM, Bidchol AM, Graul-Neumann L, Gupta A, Hehr U, Lessel D, Nader S, Shah H, Wickert J, Kutsche K. 2016. Phenotype and genotype in patients with Larsen syndrome: clinical homogeneity and allelic heterogeneity in seven patients. BMC Med Genet 17: 1-14. doi:10.1186/s12881-016-0290-6

Kelly BJ, Fitch JR, Hu Y, Corsmeier DJ, Zhong H, Wetzel AN, Nordquist RD, Newsom DL, White P. 2015. Churchill: an ultra-fast, deterministic, highly scalable and balanced parallelization strategy for the discovery of human genetic variation in clinical and population-scale genomics. Genome Biol 16: 1-14. doi:10.1186/ s13059-014-0577-x

Koboldt DC, Mihalic Mosher T, Kelly BJ, Sites E, Bartholomew D, Hickey SE, McBride K, Wilson RK, White P. 2018. A de novo nonsense mutation in ASXL3 shared by siblings with Bainbridge-Ropers syndrome. Cold Spring Harb Mol Case Stud 4: 1-6. doi:10.1101/mcs.a002410 
Krakow D, Robertson SP, King LM, Morgan T, Sebald ET, Bertolotto C, Wachsmann-Hogiu S, Acuna D, Shapiro SS, Takafuta T, et al. 2004. Mutations in the gene encoding filamin B disrupt vertebral segmentation, joint formation and skeletogenesis. Nat Genet 36: 405-410. doi:10.1038/ng1319

Larsen LJ, Schottstaedt ER, Bost FC. 1950. Multiple congenital dislocations associated with characteristic facial abnormality. J Pediatr 37: 574-581. doi:10.1016/S0022-3476(50)80268-8

Lu J, Lian G, Lenkinski R, De Grand A, Vaid RR, Bryce T, Stasenko M, Boskey A, Walsh C, Sheen V. 2007. Filamin B mutations cause chondrocyte defects in skeletal development. Hum Mol Genet 16: 1661-1675. doi:10 $.1093 / \mathrm{hmg} / \mathrm{ddm} 114$

Robertson S. 2017. FLNB-related disorders. In GeneReviews (ed. Adam MP, Ardinger HH, Pagon RA, et al.), pp. 1-19. University of Washington, Seattle.

Sawyer GM, Clark AR, Robertson SP, Sutherland-Smith AJ. 2009. Disease-associated substitutions in the filamin $B$ actin binding domain confer enhanced actin binding affinity in the absence of major structural disturbance: insights from the crystal structures of filamin B actin binding domains. J Mol Biol 390: 1030-1047. doi:10.1016/j.jmb.2009.06.009

Stossel TP, Condeelis J, Cooley L, Hartwig JH, Noegel A, Schleicher M, Shapiro SS. 2001. Filamins as integrator of cell mechanics and signalling. Nat Rev Mol Cell Biol 2: 138-145. doi:10.1038/35052082

Zhou X, Tian F, Sandzén J, Cao R, Flaberg E, Szekely L, Cao Y, Ohlsson C, Bergo MO, Borén J, et al. 2007. Filamin B deficiency in mice results in skeletal malformations and impaired microvascular development. Proc Natl Acad Sci 104: 3919-3924. doi:10.1073/pnas.0608360104 


\section{COLD SPRING HARBOR Molecular Case Studies}

\section{Novel in-frame FLNB deletion causes Larsen syndrome in a three-generation pedigree}

Scott E. Hickey, Daniel C. Koboldt, Theresa Mihalic Mosher, et al.

Cold Spring Harb Mol Case Stud 2019, 5: a004176

Access the most recent version at doi: $10.1101 / \mathrm{mcs} . a 004176$

\section{Supplementary http://molecularcasestudies.cshlp.org/content/suppl/2019/12/12/mcs.a004176.D Material C1}

References This article cites 11 articles, 3 of which can be accessed free at: http://molecularcasestudies.cshlp.org/content/5/6/a004176.full.html\#ref-list-1

License This article is distributed under the terms of the Creative Commons Attribution-NonCommercial License, which permits reuse and redistribution, except for commercial purposes, provided that the original author and source are credited.

Email Alerting Receive free email alerts when new articles cite this article - sign up in the box at the Service top right corner of the article or click here. 